\title{
Fertility Domain
}

National Cancer Institute

\section{Source}

National Cancer Institute. Fertility Domain. NCI Thesaurus. Code C95089.

A subject domain utilized for the submission of information encompassing and representing data, vocabulary or records related to fertility. 